\title{
Autonomic Adaptations mediate the effect of Hydration on Brain Functioning, Cognition and Mood: A Randomized Controlled Trial.
}

\author{
$20^{\text {th }}$ July 2018 \\ Hayley A Young, Stephen Johnston, John M Fletcher and David Benton \\ Department of Psychology, Swansea University, Swansea SA2 8PP,Wales, UK \\ * PepsiCo, 100 Summit Lake Drive, Valhalla NY, USA
}

Phone ++44 1792295607

Fax ++44 1792295679

email h.a.young@swansea.ac.uk 


\section{ABSTRACT}

Dehydration (water loss $>2.0 \%$ of body weight) has significant negative effects on physical and mental performance. In the current study, the effects of minor (water loss $<1.0 \%$ of body weight) hypo-hydration on CNS function, mood and cardiovascular function were measured. On two mornings twelve male participants were exposed to a temperature of $30 \mathrm{oC}$ for four hours and either did or did not drink two $150 \mathrm{ml}$ glasses of water during that time. Subjects not given water lost $0.6 \%$ body weight. Subjects participated in a fMRI protocol during which they completed a modified version of the Paced Auditory Serial Addition Test (PASAT): a challenging arithmetic task designed to elicit autonomic arousal. Throughout the morning the subjects had their heart rate variability (HRV) monitored. At periodic intervals, subjects rated their thirst and mood by visual analogue scales and at the end of the PASAT task, they rated its difficulty. Changes in body temperature and fluid loss (perspiration and urine) and urine osmolality were monitored. Decreases in functional magnetic resonance imaging (fMRI) blood oxygen dependent level (BOLD) activity in the orbitofrontal cortex, ventral cingulate gyrus, dorsal cingulate cortex, hypothalamus, amygdala, right striatum, post-central gyrus and superior parietal cortex were seen when participants were hypo-hydrated. These deactivations were associated with reduced HRV, greater perceived effort and more anxiety. Drinking water also prevented a decline in memory and mood. These data are discussed in relation to a model that describes how autonomic regulatory and interoceptive processes may contribute to the cognitive and affective consequences of hypo-hydration. This trial was registered at clinicaltrials.gov NCT03525470. 


\section{INTRODUCTION}

The adverse consequences of dehydration (water loss $\geq 2.0 \%$ of body mass) have been well described and include declines in physical and mental performance and increased blood pressure and heart rate ${ }^{1}$. In healthy individuals such large changes in hydration status are rare and are associated with water deprivation and prolonged exposure to hot environmental temperatures and/or prolonged exercise. It is often assumed that thirst and homoeostatic mechanisms keep within a narrow range the hydration status of those with access to water and living in temperate environments. Furthermore, it has been assumed that small variations in hydration status, in what might be described as the normal day to day range, have no significant influence on higher mental functions such as cognition and mood ${ }^{2}$. Recent studies have, however, shown that some aspects of cognition and brain function are adversely influenced by small degrees of dehydration. In an experimental protocol that tested performance on a simulated driving task, hypo-hydration (loss of $1.1 \%$ body mass) caused increased errors and changes in EEG alpha and theta waves indicative of drowsiness ${ }^{3}$.

The physiological regulation of fluid balance is relatively well understood ${ }^{4}$, several forebrain and brainstem circuitries interact with peripheral neural and humoral signals to collaboratively maintain the osmolality and volume of fluids. One counterregulatory mechanism involves changes in cardiovascular and autonomic functioning. Hypo-hydration induces cardiovascular strain ${ }^{1}$ : for every $1 \%$ decrease in body mass during exercise there is an increase in heart rate of 3.29 beats per min (bpm) ${ }^{5}$. Dehydration decreases the volume of blood (absolute hypovolemia), reducing cardiac output and necessitating an increase in heart rate to maintain blood 
pressure. When there is a simultaneous elevation in skin temperature blood vessels are dilated resulting in relative hypovolemia, an inadequate distribution of blood volume between the periphery and core. During exercise these mechanisms have the potential to compromise global cerebral perfusion ${ }^{6}$; however, hydration status may also modulate vascular responses during both orthostatic and psychological stress. For instance, Schroeder et al ${ }^{7}$ found that drinking water was associated with a higher orthostatic tolerance, suggesting that cerebral blood flow may be improved after fluid consumption. In addition, Rochette and Patterson ${ }^{8}$ found that during a stressful task, when females were better hydrated, they had a higher stroke volume and lower total peripheral resistance. Interestingly, even in the absence of hypohydration, water ingestion is followed by an increase in cardiac vagal control $^{9}$; an effect thought to counteract the pressor effects of sympathetic activation. One aim of the present study was to determine, by monitoring heart rate variability (HRV), the effect on cardiac vagal activity of mild hypo-hydration at a level that would be likely to occur on a day to day basis.

The neuro-visceral underpinnings of cardiac control have begun to be elucidated. Essentially the prefrontal cortex exerts inhibitory GABAergic control over the limbic regions of the brain that ultimately control heart rate ${ }^{10,11}$. This implies that in the presence of a physiological or psychological stressor that necessitates an increase in heart rate, there will be an associated decline in prefrontal neural activity. The relevance is that individual differences in HRV predict mood and cognitive performance, especially in tasks associated with the prefrontal and cingulate cortices 12. 
Given this background it is plausible that cardiovascular and autonomic adaptations, associated with hypo-hydration, may have cognitive and/or affective consequences. The present study reports, for the first time, that change in blood flow to certain neural systems as indicated by fMRI can be observed with as little as a $0.6 \%$ decrease in body mass. It was shown that increases in osmolality, and decreases in body mass, resulted in changed activity in the blood flow to the ventral cingulate gyrus ( $\mathrm{vCG}$ ) and medial orbito-frontal cortex (mOFC) that reflected autonomic adaptations to the physiological challenge of hypo-hydration. In addition we show here that these adaptations resulted in cognitive and affective consequences.

\section{STATISTICAL ANALYSIS}

\section{Hydration indices}

Fluid loss due to perspiration and breathing (hereafter referred to as perspiration rate) was estimated as the percentage change in body mass from baseline to the end of the session prior to urination (Figure 1). Total body mass lost, including urination, was calculated as total percentage change in body weight. For body temperature and osmolality change scores were calculated (end of the morning minus baseline). Similarly, thirst change scores were calculated (end of morning minus thirst following the standard breakfast).

\section{Memory, Attention and Mood outside the scanner}

For each cognitive measure (described in the methods section) change scores were calculated (end of the morning minus baseline) and analysed using appropriate repeated measures ANOVA designs (SPSS version 22). 


\section{Heart rate variability}

Interbeat (RR) intervals were recorded at rest at the start of the procedure and again at the end. Interbeat interval data were analysed using Kubios HRV Analysis Software $2.0^{13}$ (The Biomedical Signal and Medical Imaging Analysis Group, Department of Applied Physics, University of Kuopio, Finland). Data were visually inspected for artefacts caused by ectopic beats, poor conductivity etc. A very low correction threshold was chosen for artefact correction ( 0.45 from local average) so not to distort natural variability. Less than $1 \%$ of beats were identified as artefacts. Time domain HRV indices included mean R-R interval (a measure of basic heart rate), the standard deviation of normal to normal R-R interval (SDNN) (measures total variability in the series) and the root mean square of the standard deviation (RMSSD) (a measure of parasympathetic nervous system activity). To determine the effect of hydration, change scores were calculated (end of the morning minus baseline) for each index.

\section{fMRI analysis}

BOLD sensitive echo planar images were collected as participants performed the arithmetic task $(\mathrm{TE}=30 \mathrm{~ms}, \mathrm{TR}=3000 \mathrm{~ms}$, flip angle $=90$, axial plane, interleaved acquisition, voxels $=3 \mathrm{~mm} 3 \mathrm{~mm} 3 \mathrm{~mm}$, slices $=44$ ). In each of the three separate runs a total of 135 volumes were collected. Additionally a high-resolution magnetization-prepared rapid acquisition with gradient echo (MPRAGE) 3D volume image was acquired for each participant $(\mathrm{TE}=4.82 \mathrm{~ms}, \mathrm{TR}=2500 \mathrm{~ms}$, flip angle $=$ 7 , sagittal plane, $1 \mathrm{~mm}^{3}$ isotropic voxels, 179 slices). 
Analyses were conducted using BrainVoyager QX $2.8^{14}$ (Brain Innovations B.V., the Netherlands). Data were pre-processed as follows: 3D motion correction (6 degrees of freedom) with sinc interpolation to account for participant head movement during the scanning procedure, linear trend removal and temporal high pass filtering (low cutoff: 3 cycles per run), and spatial smoothing using a $6 \mathrm{~mm}$ Gaussian FWHM filter. Data were normalised to the standard Talairach space ${ }^{15}$ using sinc interpolation prior to group analysis. The group analysis involved estimating task-correlated activity using a general linear model (GLM) approach. The predictors for the GLM were created by convolving the timecourse of the stimulation periods (arithmetic operation) with a canonical HRF response. Each participants beta estimates, obtained via the GLM, were then input into a voxel wise second level random effects analysis. The threshold set for statistical significant was a voxel-wise $p<.05$, with an additional multiple comparison correction applied via cluster level thresholding using the Monte Carlo simulation tool implemented in BrainVoyager QX 2.8 (also with a statistical acceptance threshold of $p<.05)$.

\section{ASL analysis}

For each participant an arterial spin labelling (ASL) sequence was run after the functional imaging experiment had been completed. The ASL sequence parameters were $\mathrm{TR}=2500 \mathrm{~ms}, \mathrm{TE}=12 \mathrm{~ms}$, in-plane resolution $=4 \mathrm{~mm} \times 4 \mathrm{~mm}, 90$ averages for each label and control pair, slice thickness $=8 \mathrm{~mm}$ with a $2 \mathrm{~mm}$ gap, 9 slices were collected. The labelling time was $700 \mathrm{~ms}$ and the post-labelling time was $1800 \mathrm{~ms}$. The labelling plane was placed $18.8 \mathrm{~mm}$ inferior to the bottom slice. Scans were planned such that the ASL were in an AC-PC orientation, with the topmost scan aligned with the most superior aspect of the participant's brain. ASL data were 3D 
motion corrected and normalised to Talairach space. Relative perfusion values were obtained for each participant and condition using the ASL toolbox implemented in BrainVoyager QX 2.8. Significant differences between dehydrated and water conditions were assessed using paired t-test of the relative perfusion values.

\section{RESULTS}

\section{Hydration indices}

The effect of drinking on percentage total weight loss, perspiration rate and changes in osmolality, thirst and body temperature were analysed using RMANOVA. As expected when participants did not drink water they lost more weight both before $(F(1,11)=214.853, p<0.0001)$ and after $(F(1,11)=34.777, p<0.0001)$ urination; in total an average of $0.6 \%$ of their body mass (Table 1 ). Hypo-hydration resulted in a significant increase in urine osmolality $(F(1,11)=23.89, p<0.001)$ and participants tended to be more thirsty when they did not drink $(F(1,11)=4.178, p<0.07)$. There was no difference in participants body temperature depending on whether or not they consumed water $(F(1,11)=1.887, p=.197)$.

\section{Memory, Attention and Mood outside the scanner}

Drinking prevented a decline in immediate memory which was observed when no water had been consumed $(F(1,11)=5.898, p<0.03$; Table 1). A similar pattern occurred for delayed memory although this effect just failed to reach significance $(F(1,11)=2.581, p<0.1)$. Participants tended to perform the arrow flankers task more quickly $(F(1,11)=2.581, p<0.1)$ and more accurately $(F(1,11)=0.642, p<0.4)$ if they had drunk, although these effects were not significant (Table 1). Similarly, there were no differences in RT on the serial sevens task depending on whether they 
drunk $(F(1,11)=2.477, p<0.1)$ but participants tended to make fewer errors $(F(1,11)$ $=4.304, p<0.06)$. Overall participants mood declined significantly when they did not drink water; an effect prevented by drinking $(F(1,11)=9.665, p<0.01)$.

\section{Heart rate variability}

The effect of drinking water on heart rate variability was considered using RMANOVA (Table 1; Figure 2). When participants consumed water they had a larger increase in their average R-R interval, that is they had a lower heart rate $(F(1,11)=5.766, p<0.03)$. In addition, when participants had drunk water they had higher heart rate variability as shown by a larger standard deviation of the R-R interval $(F(1,11)=9.120, p<0.01)$ and a larger root mean squared of standard deviation of $R-R$ interval (a measure of vagal activity) $(F(1,11)=6.128, p<0.03)$.

\section{Performance in the scanner}

Data were analysed using two (Water, No water) $X$ three (block 1 , block 2 , block 3 ) RMANOVA. Main effects are shown in Table 2. The number of non-responses $(F(1,11)=.277, p=.609)$, reaction times $(F(1,11)=1.884, p=.197)$ and accuracy $(\mathrm{F}(1,11)=.549, \mathrm{p}=.474)$ did not depend on whether participants had drunk (Table 2).

\section{Ratings of difficulty and mood in the scanner}

The subjective responses were analysed using RMANOVA with condition (Water, No water) and block $(1,2,3)$ as repeated measures factors. There were no effects of drinking on ratings of anxiety $(F(1,11)=.693, p=.427)$, happiness $(F(1,11)=1.875$, 
$\mathrm{p}=.204)$, energy $(F(1,11)=2.047, \mathrm{p}=.186)$ or difficulty $(F(1,11)=.123, \mathrm{p}=.733)$ (Table 2).

\section{Brain functioning}

\section{fMRI Results}

A comparison of activation during the arithmetic task in the water vs. the dehydration condition revealed several clusters that achieved statistical significance. A large cluster was centred on the ventral cingulate gyrus (Talairach co-ordinate: 4, 14, -6; Figure 4), that extended into both the hypothalamus and striatal brain regions. Specifically, striatal activity was observed in the bilateral caudate nucleus and putamen. The same cluster also spread inferiorly to right amygdala. To the anterior aspect the activity spreads into medial orbito-frontal cortex. A second small cluster was observed in the dorsal cingulate gyrus (Talairach co-ordinate: 9, 23, 20). The final cluster of activity was seen in left post-central gyrus ('PoCG', Talairach coordinate: $-36,-19,-35$; Figure 3) and extended superior and anterior into the superior parietal cortex. There were no significant areas of activation for the reverse contrast of dehydration > water.

\section{Cerebral blood flow}

Analysis of the ASL data did not reveal any significant differences in resting perfusion for either the contrast of dehydration vs. water, or water vs. dehydration.

\section{Association between differences in brain activity, HRV and hydration indices}

Peaks of activity occurred on the ventral cingulate gyrus (vCG), the border of the medial orbitofrontal cortex (mOFC) and in the left post-central gyrus (PoCG). To 
investigate the possible mechanisms behind the observed differences in brain (see above), the difference in activity between when subjects had or had not consumed water was correlated (Pearson's r) with difference scores (no water minus water) for the total percentage of weight lost, perspiration rate and changes in temperature, osmolality, heart rate variability and thirst. Results are displayed in Table 3. Only thirst correlated with activity in the PoCG $(r=-.49, p<0.05$; Figure 3$)$; a greater thirst in the no water condition was associated with lower activity. The perspiration rate correlated with all three regions $(r=.58, \mathrm{p}<0.001$ for mOFC, $r=.55, \mathrm{p}<0.05$ for vCG, $r=.54, p<0.05$ for PoCG) and osmolality was associated with activity in the mOFC and $\mathrm{vCG}$ areas $(r=-.85, \mathrm{p}<0.002$ for mOFC and $r=.57, \mathrm{p}<0.05$ for $\mathrm{vCG}$; Figure 4). In addition, total weight loss, including the amount urinated, was also associated with lower activity in the $\mathrm{vCG}(r=.58, \mathrm{p}<0.05)$. The general pattern was that when water was not given, the more weight that was lost, and the higher osmolality (they were more hypo-hydrated), the lower the brain activity. Heart rate variability correlated significantly with activity in the mOFC $(r=.78, p<0.005)$ and vCG areas $(r=.77, p<0.05$; Figure 4); in both instances a smaller increase in $R-R$ interval (i.e a higher heart rate) was associated with lower activity brain activity when no water had been drunk. Similar effects were found for the HRV indices; both the SDNN and RMSSD correlated significantly with activity in the mOFC $(r=.42, p<0.05$ for SDNN and $r=.48, \mathrm{p}<0.05$ for RMSSD) and vCG $(r=58, \mathrm{p}<0.05$ for mOFC and $r=.52, \mathrm{p}<0.05$ for $\mathrm{vCG}$ ) regions, indicating that the hypo-hydration-associated decline in activity may reflect changes in cardiac vagal activity. Overall these findings suggested that the decline in brain activation that was observed when participants didn't drink reflected autonomic responses to the low-level physiological challenge of hypo-hydration. 


\section{Association between differences in activation and behaviour}

As noted above, in the scanner there were no differences in performance or mood depending upon whether participants had consumed water. As such it is not clear whether the observed changes in autonomic and brain functioning have cognitive or affective consequences or whether they are independent mechanisms. Therefore to establish whether the observed differences in brain activity, heart rate and hydration indices were related to cognition/mood they were correlated (Pearson's r) with performance differences from the arithmetic task (no water minus water) and ratings of perceived difficulty and mood. Data are presented in Table 4. The average length of the R-R interval correlated with both anxiety $(r=-.61, p<0.03)$ and perceived difficulty $(r=-.45, p=0.06)$; a lower $R R$ interval (i.e higher heart rate) in the no water condition was related to greater anxiety and more perceived effort (Figure 4). Similar associations were found between the subjective ratings and activity in the mOFC ( $r=$ $-.72, p<0.01$ for anxiety; $r=-.60, p<0.02$ for difficulty) and between the subjective ratings and activity in the vCG $(r=-.77, p<0.007$ for anxiety; $r=-.75, p<0.004$ for difficulty); in each case lower activity was associated with more anxiety and greater difficulty. Lower activity in the VCG in the no water condition was also associated with slower reaction times $(r=-.51, p<0.05)$. Osmolality was related to ratings of energy $(r=-.45, p<0.05)$, depression $(r=-.56, p<0.05)$ and anxiety $(r=-.54, p<$ $0.05)$ whilst in the scanner; those with the greatest increase in osmolality in the no water condition reported being more anxious and feeling less energetic and happy. The number of non- responses to the arithmetic task correlated with both thirst $(r=$ $.66, p<0.02)$ and activity in the PoCG $(r=.57, p<0.05)$; a greater number of nonresponses in the no water condition was related to having more thirst and lower 
activity in the PoCG (Figure 3). Temperature, thirst and amount of weight lost before and after urination were not associated with any aspect of performance (Table 4).

\section{Associations with performance and mood outside the scanner}

Correlations between regional brain activation, HR and hydration parameters, and behavioural performance and mood outside the scanner, are available as supplementary information (S1). Mood again correlated with the average length of the RR interval $(r=.42, p<0.05)$, SD of the RR interval $(r=.62, p<0.01)$, the $\operatorname{RMSDD}(r=.62, p<0.01)$ and activity in the striatum $(r=.47, p<0.05)$; a poorer mood when subjects did not drink was associated with reduced cardiac vagal activity and lower striatal activity. Losing more weight was also associated with a poorer $\operatorname{mood}(r=.44, p<0.05)$. Interestingly, in this analysis mood was also associated with thirst; the greater the thirst the poorer the $\operatorname{mood}(r=-.733, p<0.008)$. Similarly, being more thirsty was associated with a greater decline in both immediate $(r=-$ $.641, \mathrm{p}<0.02)$ and delayed $(r=-.710, p<0.01)$ episodic memory. A decline in activity in the mOFC was associated with having poorer RT on the working memory task; a decline in activity in the PoCG and VCG was associated with making more errors on the test of focused attention and having poorer immediate episodic memory (Table S1).

\section{DISCUSSION}

The present study reports that hypo-hydration adversely influenced brain function. As a result of not drinking water during the experimental protocol, task related activity in the autonomic network of the brain was reduced; specifically activity in the orbito-frontal cortex, ventral cingulate gyrus, dorsal cingulate cortex, hypothalamus, 
amygdala, right striatum, post-central gyrus and superior parietal cortex was affected. When subjects were hypo-hydrated they had a higher HR and lower HRV; effects that correlated with peak activation in the vCG and mOFC. Importantly, these effects were associated with poorer mood and cognition. These observations are consistent with the hypothesis that hypo-hydration elicits a change in autonomic regulatory activity and brain function with potential adverse consequences for mood and cognition.

In the context of exercise severe hypo-hydration ( $>2 \%$ loss of body weight) elevates $\mathrm{HR}^{2}$ but there has been little study of the effect of everyday fluctuations in hydration status on cardiac function. The present study found that in subjects at rest hypohydration resulted in a shorter average R-R interval i.e a higher HR (on average 5.8 beats per minute higher) and lower HRV (Figure 2). Interestingly, limited evidence suggests that drinking water, even in the absence of hypo-hydration, may modulate the cardiac vagal response; 20 and 25 min after drinking $500 \mathrm{ml}$ of water heart rate fell from 67 to $60 \mathrm{bpm}$ and RMSSD increased by $13 \mathrm{~ms}^{9}$. This latter finding is important because it might help explain how acute water supplementation can benefit mood irrespective of hydration status ${ }^{16}$.

Areas of the brain mediating sympathetic and parasympathetic control of the ANS include regions of the limbic forebrain such as the subgenual and pregenual cingulate, amygdala/ventral striatum and medial prefrontal cortex ${ }^{11}$ : as can be seen in Figure 5, change in many of the same regions were associated with hypohydration. Mathews et al. ${ }^{17}$ studied the association between activity in the vCG and peak high frequency power (a HRV index of vagal activity) during a Stroop task; they 
found a positive association such that greater activity in the VCG was associated with a higher vagal tone. Similarly in the present study, having higher activity in the vCG and mOFC was associated with greater parasympathetic activity (indexed by the average RR interval, SDRR and RMSSD). These findings indicate that the hydration related differences in brain activity in these regions may be the result of hydration induced autonomic modulation. Interestingly, drinking water both ameliorated the task related decline in brain activity as indicated by $\mathrm{fMRI}$ and increased HRV. A novel hypothesis is that hypo-hydration induces increased cardiovascular strain, which in turn necessitates a decline in activity in the autonomic network of the brain; effects that could compromise cognitive and affective processing.

Data from our laboratory have shown that even in a healthy population lower HRV is associated with poorer mood and cognition and higher perceived stress ${ }^{12}$. The present study found that a higher heart rate and lower HRV were associated with increases in anxiety and perceived effort during scanning; these effects were also associated with having a greater deactivation in the mOFC and vCG. In addition, a greater deactivation in the VCG was associated with performing the arithmetic task more slowly whereas those with a higher HRV performed the task more accurately. Hypo-activation of the anterior cingulate and frontal cortices is common in a range of anxiety disorders ${ }^{18}$ and during induced anxiety ${ }^{19}$. In addition, interventions known to reduce anxiety increase activity in these regions ${ }^{20}$; it is therefore plausible that the benefits of drinking water reflect similar mechanisms.

Notably, scales taken whilst participants were in the scanner did not differ according to whether or not they consumed water (Table 2), however, before they left the $30^{\circ} \mathrm{C}$ 
environment (see methods section) there were between condition differences in mood and cognition. Temperatures in excess of $27^{\circ} \mathrm{C}$ exacerbate the effects of dehydration, induce peripheral vasodilatation and result in relative hypovolemia, increasing cardiac strain ${ }^{2}$. Thus autonomic adaptations that mediate the effects of hydration are likely to be enhanced by a warm environment and it is plausible that by the time participants entered the scanner (on average $22^{\circ} \mathrm{C}$ ) the exacerbating effect of heat would have been diminished. Supporting this view, an increase in body temperature was associated with poorer mood and making more errors on the working memory task whilst in the warm environment; although no relationship was found between body temperature and performance or mood VAS ratings once participants had moved to the scanner. Interestingly, Farrell et al. ${ }^{21}$ recently reported that regions of the central autonomic network were activated in response to both thermogenic and psychogenic sweating events, in particular the anterior cingulate cortex, lentiform nuclei and parietal cortex. In the present study perspiration rate correlated significantly with activity in all three regions of difference; the mOFC, vCG and PoCG. If such regions are also involved in hypo-hydration associated cognitive and affective deficits, this might help to explain the effects of the interaction between thermoregulation and hypo-hydration on cognition and mood.

Interestingly it has been argued that because the cingulate cortex has been associated with a wide range of cognitive challenges it is likely that it mediates a more general autonomic response; one that reflects the integration of internally and externally generated demands ${ }^{22}$. The present findings are consistent with this hypothesis; activity in this region was not only associated with changes in HRV but also osmolality and perspiration. It is plausible that the integration of afferent 
interoceptive information from both humeral and neuronal signalling may be integrated in the anterior cingulate (ACC) which in turn orchestrates an appropriate counter-regulatory response.

Besides changes in ANS activity, thirst is an interoceptive signal that may disrupt functioning. In the brain, changes in plasma osmolality are detected by circumventricular organs such as the organum vasculosum laminae terminalis (OVLT) which have projections to the $\mathrm{ACC}^{23}$, where the conscious experience of thirst is perceived ${ }^{24,25}$. We previously reported that a loss of fluid associated with less than $1 \%$ decrease in body mass was associated with a decline in cognition; an effect prevented by drinking and mediated by thirst ${ }^{26}$. Thirst has also been associated with parietal activation, in particular the post-central gyrus ${ }^{24}$, a finding confirmed in the present study; significant differences between the drinking conditions occurred in this region of the brain and they correlated with differences in thirst (Figure 3). Notably, the activity in this complex extended towards the superior parietal cortex, a region known to be associated with arithmetic performance; activity in this region was associated with a larger number of non-responses on the arithmetic task. Understanding the potential overlap between interoceptive and cognitive networks may help illuminate the means by which hydration and other dietary components influence cognition.

Although neuroimaging studies have attempted to understand our experience of thirst, only one study has considered the effect of hydration on brain functioning during a cognitive task. Kempton et al. ${ }^{27}$ studied the effects of fluid restriction during a thermal exercise protocol on fMRI BOLD responses to the Tower of London 
task; a task of executive functioning. Dehydration did not change the ability to perform the task; however, when dehydrated there was a greater BOLD response in the fronto-parietal cortex. It was suggested that dehydration had placed demands on the brain such that greater resources were required to achieve the same level of output. If this explanation was accurate participants will find the task more difficult when hypo-hydrated but, as ratings of task difficulty were not obtained, the explanation was speculative. In the present study ratings of perceived effort were associated with declines in brain activity, albeit in different regions of the brain; there was no association between difficulty ratings and activity in the parietal cortex.

An alternative hypothesis might be that, rather than altering neural activity, hypohydration reduces cerebrovascular coupling. Given that brain functional imaging methods, such as fMRI, are sensitive to changes in cerebral blood flow (CBF) it is plausible that the observed differences in regional BOLD activity might reflect differences in CBF. Hypo-hydration of $1.3 \%$ reduced mean middle cerebral artery blood flow velocity during a cold pressor test, suggesting that the cerebrovascular response to acute stressful stimuli may be altered by hydration status ${ }^{28}$. However, the present study found no effects of hydration on global or regional blood flow using resting state ASL; nonetheless, it remains possible that dynamic cerebrovascular reactivity, rather than resting state auto-regulation, may have been compromised under the present conditions. This remains an important consideration for future research that examines the effects of hydration on brain functioning using imaging methodologies. 
In conclusion, the present study reports that when participants consumed water, compared with when they were mildly hypohydrated ( $0.6 \%$ loss in body weight) they had improved mood as indicated by VAS ratings, improved cognitive performance and increased neural activity as indicated by $F M R I$ when faced with a demanding task. For the first time we have highlighted important regulatory neural mechanisms that may account for the psychological benefits of maintaining hydration status. When water was not consumed more weight was lost, osmolality increased and HRV decreased; effects that predicted a larger task related deactivation in the autonomic network of the brain. Only recently have the interactions between bodily reactions and cognitive processes begun to be elucidated and such phenomena are still often treated as confounding factors. Indices of ANS function have been used as objective measures of affective states and these responses have been treated as epiphenomena, rather than intrinsic to the cognitive or emotional process. As evidenced here, it is possible that changes in ANS activity have consequences for cognition and mood. As such hypo-hydration to the extent that it influences ANS activity per se may have negative consequences for cognition and mood.

Given the prevalence of voluntary hypo-hydration these findings have important implications for vulnerable cross-sections of the population. Furthermore, the cognitive benefits of drinking may have important implications for populations, such as older adults and children ${ }^{29}$ who are at a significant risk of dehydration. The fact that mild changes in hydration affected cardiovascular functioning is also a concern; the HR of participants was on average 5.8 beats per minute lower when they had consumed water. Given that there is a progressive increase in the risk of heartdisease as resting heart rate increases ${ }^{30}$, and hyper-osmolarity predicted a 4.3 -fold 
increased risk of cardiovascular mortality ${ }^{31}$, maintaining adequate hydration may be an important factor for maintenance of cardiovascular health. In general, repeated low level physiological challenges such as hypo-hydration may overtime increase allostatic load, predisposing to possible negative health outcomes.

\section{METHODS}

\section{Sample characteristics}

Twelve healthy young males (average age 23.3 years (19-34), average BMI 25.4 (19-34)), gave their written informed consent after being screened for MRI safety. Participants were excluded if they had any health complaint that would affect cardiovascular functioning such as diabetes or hypertension. Similarly, anyone with a neuropsychological illness was also excluded as were those taking medication. All participants were right handed, non-smokers; before the start of the study they were asked to refrain from drinking alcohol for at least 24 hours and told to fast and avoid any beverages for at least twelve hours.

\section{Procedure}

On two occasions participants attended the laboratory that was heated to 30 degrees. Using a repeated measures design, in a randomly derived order, they received either two $150 \mathrm{ml}$ glasses of water or nothing (Figure 1). A random numbers table was used to determine the random allocation sequence which was implemented by DB. Participants were blind as to the nature of the experiement. Upon arriving at the laboratory participants were asked to provide a urine sample and were asked to completely empty their bladder, following which they were weighed and their body temperature measured. They were then fitted with a RS800 
Polar heart rate monitor electrode transmitter belt (T61) and a Polar RS800 HR monitor (Polar Electro, Kempele, Finland) that was used to collect interbeat interval measurements at a sampling rate of $1000 \mathrm{~Hz}$, while participants rested in a seated position. This instrument has been previously validated for the accurate measurement of R-R intervals and for analysing Heart Rate Variability (HRV) ${ }^{32}$. Participants were then provided with a standard breakfast consisting of $50 \mathrm{~g}$ of Quaker Oat So Simple Original Porridge (187kcal, $2.9 \mathrm{~g}$ fat, $10.6 \mathrm{~g}$ sugar, $0.11 \mathrm{~g}$ salt) plus $150 \mathrm{ml}$ of either decaffeinated tea or coffee. After breakfast participants were weighted again, completed the cognitive test battery (see below) and rated their mood and thirst. After this they were allowed to rest while either watching TV or reading. Three hours later participants were asked to provide another urine sample and again had their HRV, body temperature and weight measured, completed the test battery and rated their mood and thirst. Finally they were escorted to the MRI laboratory for the scanning procedure. The procedure was approved by Swansea University ethics committee (ref: 01.03.2015.1) and carried out in accordance with the principles laid down by the declaration of Helsinki 2013. All participants completed the study. This trial was registered at clinicaltrials.gov NCT03525470 $(15 / 05 / 18)$.

\section{Behavioural test battery}

Immediate and delayed episodic memory

Episodic memory was measured by recalling a list of 30 words. Using the MRC Psycholinguistic Database, words were matched for the number of syllables, imageability and the frequency with which they occur in English. Using a recorder words were aurally presented one every $2 \mathrm{~s}$ and immediately after the presentation as 
many words as possible were written down (immediate recall). Approximately 20 min later, after completion of the other tasks, subjects again recalled the words (delayed recall).

\section{Working memory}

Working memory was assessed using a modified version of the serial sevens test which involved presenting a participant with a starting number from which they must subtract 7. A computerised version of the test was implemented. A randomly generated starting number between 800 and 999 was displayed on a monitor for two seconds. Participants were required to indicate by pressing one of two keys, that corresponded to yes or no, whether a second number was, or was not, exactly seven less. Two seconds after pressing the button the next trial started and a total of twenty-eight trials were completed. The test was scored as the average of the time taken, in milliseconds, to perform a subtraction and the number of errors made.

\section{Focused attention}

A modified version of the Eriksen and Eriksen ${ }^{33}$ flanker task was used to measure focused attention. The Arrow Flankers test measures the ability to direct attention and ignore peripheral information. Participants were required to indicate whether the middle arrow was pointing to the right or left by pressing the corresponding arrow on the keyboard. Either side of the central arrow were distractors. The flanking pairs of symbols could be squares ( $\square \square<\square \square$ ), congruent arrows (pointing in the same direction $(\gg>\gg))$ ) or incongruent arrows (pointing in the opposite direction $(<<><<)$ ). A stimulus remained on screen for 1.8 seconds or until the key press was registered. There was a randomly varying inter-stimulus interval of between 1 and 3 seconds, on 
average 2 seconds. Seventy stimuli were presented pseudo-randomly with congruent, incongruent or neutral stimuli appearing on twenty occasions. The test was scored as the average response time in milliseconds and the number of incorrect responses.

\section{Mood}

Subject were asked to report on visual analogue scales how they felt "at this moment" using visual analogue scales with pairs of adjectives at the ends of $100 \mathrm{~mm}$ lines; Composed/Anxious; Hostile/Agreeable; Elated/Depressed; Unsure/Confident; Energetic/Tired; Confused/Clearheaded as described by McNair and Lorr ${ }^{34}$. Mood was measured at the end of each test battery.

\section{Thirst}

Participants were asked to responds to the question "how thirsty are you feeling right now" on a single $100 \mathrm{~mm}$ visual analogue scale anchored by "Not at all" and "Extremely".

\section{Osmolality}

The osmolality of urine was assessed using an Osmomat 3000 freezing point osmometer (Gonotec $\mathrm{GmbH}$, Berlin, Germany).

\section{Body temperature}

Body temperature was measured using a TH8 Infrared Ear Thermometer (Radiant Innovation, Taiwan). 


\section{Body mass}

Body mass was measured using an electronic scale (Kern KMS-TM, Kenr and Sohn $\mathrm{GmbH}$, Germany) that, to avoid problems associated with movement, took 50 assessments over a 5 second period and produced an average value. It was sensitive enough to weigh to within 5 grams (17\% of an ounce) and could pick up over short periods changes in body mass due to breathing and perspiration. Subjects were weighed on arrival, both before and after breakfast, and again at the end, both before and after urination.

\section{Scanning procedure}

Participants were presented with an arithmetic task, modified for use in the scanner, similar to the Paced Auditory Serial Addition Task: a 'stressful' task that measures calculation ability and is known to elicit an autonomic response ${ }^{35}$. Pairs of two digit numbers appeared on a screen in red and participants were required to mentally add or subtract the numbers. After 2 seconds the screens was removed and a second screen appeared containing a correct and an incorrect answer. Participants were required to press either a left or right button to indicate which answer was correct (Figure 6). The speed of presentation is designed to be just at the level that it is possible to perform the task although it required mental effort and was 'stressful'. This allowed investigation of areas of the brain associated with working memory but also those associated with emotional arousal. Data analysed from the task were number of missed responses, the number of correct responses and average reaction time in milliseconds (on the trials that participants responded). Three blocks of four minutes were performed. At the end of each block four visual analogue scales appeared and participants were asked to rate, using the left and right key to move a 
cursor, how difficult they found the proceeding block and how happy, energetic and anxious they felt at that moment. The visual analogue scales were rated on a scale of 1-100.

\section{ACKNOWLEDGEMENT}

This research was part funded by PepsiCo Inc. JMF is an employee of PepsiCo Inc. The authors have no other conflicts of interest to declare. HY, DB, SJ, and JMF designed the research. HY and SJ analysed the data. HY, DB, SJ and JMF prepared and edited the manuscript. HY and SJ conducted the research. The views expressed in this manuscript are those of the authors and do not necessarily reflect the position or policy of PepsiCo Inc. The authors would like to thank Heather Watkins and Nadine Garland for their assistance in collecting and inputting data for this study.

\section{REFERENCES}

1 Benton, D. Dehydration influences mood and cognition: a plausible hypothesis? Nutrients 3, 555-573 (2011).

2 Sawka, M. N., Cheuvront, S. N. \& Kenefick, R. W. Hypohydration and Human Performance: Impact of Environment and Physiological Mechanisms. Sports Medicine 45, 51-60 (2015).

3 Watson, P., Whale, A., Mears, S. A., Reyner, L. A. \& Maughan, R. J. Mild hypohydration increases the frequency of driver errors during a prolonged, monotonous driving task. Physiology \& behavior 147, 313-318 (2015). 
4 Tanner. The Regulation of Fluid and Electrolyte Balance. In: Rhoades, R. A. and Bell, D.R., ed. Medical Physiology Principles for Clinical Medicine. 3rd ed. Philadelphia: Lippincott Williams and Wilkins. 419-441 (2009).

5 Adams, W. M., Ferraro, E. M., Huggins, R. A. \& Casa, D. J. Influence of body mass loss on changes in heart rate during exercise in the heat: a systematic review. The Journal of Strength \& Conditioning Research 28, 2380-2389 (2014).

6 Trangmar, S. J. et al. Dehydration accelerates reductions in cerebral blood flow during prolonged exercise in the heat without compromising brain metabolism. American Journal of Physiology-Heart and Circulatory Physiology 309, H1598-H1607 (2015).

7 Schroeder, C. et al. Water drinking acutely improves orthostatic tolerance in healthy subjects. Circulation 106, 2806-2811 (2002).

8 Rochette, L. M. \& Patterson, S. M. Hydration status and cardiovascular function: effects of hydration enhancement on cardiovascular function at rest and during psychological stress. International journal of psychophysiology 56, 81-91 (2005).

9 Helen, C., Chowdhary, S., Coote, J. H. \& Townend, J. N. Cardiac vagal response to water ingestion in normal human subjects. Clinical Science 103, 157-162 (2002)

10 Beissner, F., Meissner, K., Bär, K.-J. \& Napadow, V. The autonomic brain: an activation likelihood estimation meta-analysis for central processing of autonomic function. The Journal of Neuroscience 33, 10503-10511 (2013).

11 Thayer, J. F., Åhs, F., Fredrikson, M., Sollers, J. J. \& Wager, T. D. A metaanalysis of heart rate variability and neuroimaging studies: implications for 
heart rate variability as a marker of stress and health. Neuroscience \& Biobehavioral Reviews 36, 747-756 (2012).

12 Young, H. \& Benton, D. We should be using nonlinear indices when relating heart-rate dynamics to cognition and mood. Scientific reports 5 (2015).

13 Tarvainen, M. P., Niskanen, J.-P., Lipponen, J. A., Ranta-Aho, P. O. \& Karjalainen, P. A. Kubios HRV-heart rate variability analysis software. Computer methods and programs in biomedicine 113, 210-220 (2014).

14 Goebel, R. BrainVoyager-past, present, future. Neuroimage 62, 748-756 (2012).

15 Talairach, J. \& Tournoux, P. Co-planar stereotaxic atlas of the human brain. 3-Dimensional proportional system: an approach to cerebral imaging. (1988).

16 Masento, N. A., Golightly, M., Field, D. T., Butler, L. T. \& van Reekum, C. M. Effects of hydration status on cognitive performance and mood. British Journal of Nutrition 111, 1841-1852 (2014).

17 Matthews, S. C., Paulus, M. P., Simmons, A. N., Nelesen, R. A. \& Dimsdale, J. E. Functional subdivisions within anterior cingulate cortex and their relationship to autonomic nervous system function. Neuroimage 22, 11511156 (2004).

18 Etkin, A. \& Wager, T. D. Functional neuroimaging of anxiety: a meta-analysis of emotional processing in PTSD, social anxiety disorder, and specific phobia. American Journal of Psychiatry (2007).

19 Straube, T., Schmidt, S., Weiss, T., Mentzel, H.-J. \& Miltner, W. H. Dynamic activation of the anterior cingulate cortex during anticipatory anxiety. Neuroimage 44, 975-981 (2009). 
20 Zeidan, F., Martucci, K. T., Kraft, R. A., McHaffie, J. G. \& Coghill, R. C. Neural correlates of mindfulness meditation-related anxiety relief. Social cognitive and affective neuroscience 9, 751-759 (2014).

21 Farrell, M. J., Trevaks, D., Taylor, N. A. \& McAllen, R. M. Brain stem representation of thermal and psychogenic sweating in humans. American Journal of Physiology-Regulatory, Integrative and Comparative Physiology 304, R810-R817 (2013).

22 Critchley, H. D., Wiens, S., Rotshtein, P., Öhman, A. \& Dolan, R. J. Neural systems supporting interoceptive awareness. Nature neuroscience 7, 189-195 (2004).

23 Hollis, J. H., McKinley, M. J., D'Souza, M., Kampe, J. \& Oldfield, B. J. The trajectory of sensory pathways from the lamina terminalis to the insular and cingulate cortex: a neuroanatomical framework for the generation of thirst. American Journal of Physiology-Regulatory, Integrative and Comparative Physiology 294, R1390-R1401 (2008).

24 Denton, D. et al. Correlation of regional cerebral blood flow and change of plasma sodium concentration during genesis and satiation of thirst. Proceedings of the National Academy of Sciences 96, 2532-2537 (1999).

25 Farrell, M. J. et al. Cortical activation and lamina terminalis functional connectivity during thirst and drinking in humans. American Journal of Physiology-Regulatory, Integrative and Comparative Physiology 301, R623R631 (2011).

26 Benton, D., Jenkins, K. T., Watkins, H. T. \& Young, H. A. Minor degree of hypohydration adversely influences cognition: a mediator analysis. The American Journal of Clinical Nutrition 104, 603-612 (2016). 
27 Kempton, M. J. et al. Dehydration affects brain structure and function in healthy adolescents. Human brain mapping 32, 71-79 (2011).

28 Perry, B. G., Bear, T. L., Lucas, S. J. \& Mündel, T. Mild dehydration modifies the cerebrovascular response to the cold pressor test. Experimental physiology 101, 135-142 (2016).

29 Benton, D. \& Burgess, N. The effect of the consumption of water on the memory and attention of children. Appetite 53, 143-146 (2009).

30 Fox, K. et al. Resting heart rate in cardiovascular disease. Journal of the American College of Cardiology 50, 823-830 (2007).

31 Briongos-Figuero, S. et al. blood hyperosmolarity as a predictor of mortality in patients after acute coronary syndrome: how dehydration affects the prognosis of our patients. Journal of the American College of Cardiology 61 (2013).

32 Nunan, D. et al. Validity and reliability of short-term heart-rate variability from the Polar S810. Medicine+ Science in Sports+ Exercise 41, 243 (2009).

33 Eriksen, B. A. \& Eriksen, C. W. Effects of noise letters upon the identification of a target letter in a nonsearch task. Perception \& psychophysics 16, 143149 (1974).

34 Lorr, M., McNair, D. M. \& Fisher, S. Evidence for bipolar mood states. Journal of personality assessment 46, 432-436 (1982).

35 Tanosoto, T. et al. Effects of the Paced Auditory Serial Addition Task (PASAT) with different rates on autonomic nervous system responses and self-reported levels of stress. Journal of oral rehabilitation 42, 378-385 (2015). 


\section{TABLES}

\begin{tabular}{|l|c|c|}
\hline & NO WATER & WATER \\
\hline Weight lost before urination (\%) & $-0.40(0.01)^{* *}$ & $-0.01(0.03)^{\star *}$ \\
\hline Weight lost after urination (\%) & $-0.59(0.03)^{* *}$ & $-0.26(0.05)^{\star *}$ \\
\hline$\Delta$ Osmolality (mOsm/kg) & $219.77(57.50)^{* *}$ & $-190(55.47)^{* *}$ \\
\hline$\Delta$ Temperature $\left.{ }^{\circ} \mathrm{C}\right)$ & $0.75(0.11)$ & $0.50(0.19)$ \\
\hline$\Delta$ Thirst (VAS) & $24.40(4.64)$ & $12.30(5.92)$ \\
\hline$\Delta$ R-R interval & $17.75(19.47)^{*}$ & $88.42(31.90)^{*}$ \\
\hline$\Delta$ SD of R-R interval & $12.59(10.43)^{*}$ & $-12.80(5.68)^{*}$ \\
\hline$\Delta$ RMSSD & $3.87(6.96)^{*}$ & $13.38(5.89)^{*}$ \\
\hline$\Delta$ Immediate memory & $-2.50(0.84)^{*}$ & $0.83(0.81)^{*}$ \\
\hline$\Delta$ Delayed memory & $-3.41(1.34)$ & $-0.16(1.19)$ \\
\hline$\Delta$ Attention RT & $18.41(26.09)$ & $-30.16(24.06)$ \\
\hline$\Delta$ Attention accuracy & $0.50(0.33)$ & $-0.16(0.44)$ \\
\hline$\Delta$ WM RT & $3.41(65.12)$ & $-199.91(94.30)$ \\
\hline$\Delta$ WM accuracy & $0.83(0.36)^{*}$ & $-0.66(0.51)^{*}$ \\
\hline$\Delta$ Mood & $-64.09(17.32)^{*}$ & $-6.45(17.81)^{*}$ \\
\hline
\end{tabular}

Table 1. The effect of drinking compared with not drinking on hydration indices, performance and mood outside the scanner and heart rate variability.

Data are mean (s.e.) for change scores across each morning (end of the morning minus baseline) which were then compared across conditions. Compared to when they drunk, when participants did not drink they lost more weight both before and after urination and their osmolality increased. They had a significantly greater increase in HRV (RR interval, SDNN, RMSDD) when they drunk compared to when they did not drink. Participants mood and memory declined less across the morning when they drunk water compared to when they did not drink. $\Delta$ Change across the morning, * Water vs. No water significant at $p<0.05,{ }^{* *}$ Water vs. No water significant at $p<0.001$. RR, interbeat interval, SDNN, standard deviation of normal to normal R-R interval, RMSSD, root mean square of the standard deviation, WM - working memory, RT - reaction times. 


\begin{tabular}{|l|c|c|}
\hline & NO WATER & WATER \\
\hline No of non-responses & $20.08(7.5)$ & $16.91(3.21)$ \\
\hline Reaction time (RT in ms) & $0.611(0.02)$ & $0.627(0.01)$ \\
\hline Accuracy (No correct) & $200.08(9.36)$ & $206.83(3.93)$ \\
\hline Perceived difficulty (VAS) & $181.16(12.0)$ & $175.00(12.0)$ \\
\hline Anxiety (VAS) & $103.50(14.6)$ & $90.20(17.78)$ \\
\hline Happiness (VAS) & $148.60(7.77)$ & $157.50(7.52)$ \\
\hline Energy levels (VAS) & $112.30(9.69)$ & $132.80(13.72)$ \\
\hline
\end{tabular}

Table 2. Effects of drinking compared with not drinking on mood, perceived difficulty and performance in the scanner.

Data are mean (s.e.). VAS, Visual Analogue Scale, RT, Reaction Time. 


\begin{tabular}{|l|c|c|c|}
\hline & mOFC & vCG & PoCG \\
\hline$\Delta$ RR interval & $.787^{* \star}$ & $.776^{* *}$ & .145 \\
\hline$\Delta$ SD of R-R interval & $.428^{*}$ & $.580^{* *}$ & .027 \\
\hline$\Delta$ RMSSD & $.486^{*}$ & $.529^{*}$ & .350 \\
\hline Perspiration rate (\%) & $.580^{*}$ & $.553^{*}$ & $.543^{*}$ \\
\hline Total weight loss (\%) & .280 & $.580^{*}$ & .354 \\
\hline$\Delta$ Temperature ('C) & .083 & -.077 & -.028 \\
\hline$\Delta$ Osmolality (mOsm/kg) & $-.856^{* *}$ & $-.573^{*}$ & -.404 \\
\hline$\Delta$ Thirst (VAS) & .175 & & \\
\hline
\end{tabular}

Table 3. Correlations between differences in brain activation and differences in hydration parameters, thirst and heart rate.

Data are Pearson's correlation coefficient $(r) \Delta$ Difference, ${ }^{* *} p<0.005$, ${ }^{*} p<0.05$, VAS Visual Analogue Scale, RR, interbeat interval, SDNN, standard deviation of normal to normal R-R interval, RMSSD, root mean square of the standard deviation, mOFC, medial orbitofrontal cortex, PoCG, post-central gyrus, VCG, ventral cingulate gyrus. 


\begin{tabular}{|c|c|c|c|c|c|c|c|}
\hline & $\begin{array}{c}\text { Number of } \\
\text { non- } \\
\text { responses }\end{array}$ & $\begin{array}{c}\text { Reaction } \\
\text { times }\end{array}$ & Accuracy & $\begin{array}{l}\text { Perceived } \\
\text { difficulty }\end{array}$ & Happy & Energetic & Anxious \\
\hline$\Delta \mathrm{RR}$ interval & .066 & -.307 & -.234 & $-.456^{*}$ & .086 & .145 & $-.613^{*}$ \\
\hline$\Delta \mathrm{SD}$ of $\mathbf{R}-\mathbf{R}$ interval & -.108 & -.326 & $.428^{*}$ & $-.540^{*}$ & -.068 & -.133 & $-.746^{*}$ \\
\hline$\Delta$ RMSSD & -.226 & -.063 & .322 & -.298 & .058 & -.195 & -.259 \\
\hline Perspiration rate (\%) & -.057 & -.240 & -.153 & -.222 & .293 & .152 & -.247 \\
\hline Total weight loss (\%) & -.096 & -.178 & -.010 & -.229 & .177 & .141 & .045 \\
\hline$\Delta$ Temperature $\left({ }^{\circ} \mathrm{C}\right)$ & -.367 & .037 & .265 & -.024 & .130 & .316 & .021 \\
\hline $\begin{array}{l}\Delta \text { Osmolality } \\
(\mathrm{mOsm} / \mathrm{kg})\end{array}$ & .082 & .098 & .345 & .394 & $-.561^{*}$ & $-.453^{*}$ & $.545^{*}$ \\
\hline$\Delta$ Thirst (VAS) & $.660^{*}$ & .222 & -.258 & .305 & -.234 & -.217 & .228 \\
\hline mOFC & -.017 & -.190 & .121 & $-.601^{*}$ & .278 & .247 & $-.722^{*}$ \\
\hline PoCG & $.573^{*}$ & -.045 & -.364 & -.026 & -.036 & -.034 & -.319 \\
\hline vCG & -.254 & $-.517^{*}$ & .258 & $-.754^{*}$ & -.228 & -.296 & $-.774^{*}$ \\
\hline
\end{tabular}

Table 4. Correlations between differences in brain activation, hydration parameters and performance and difficulty ratings in the scanner.

${ }^{*} p<0.05 R R$, interbeat interval, SDNN, standard deviation of normal to normal R-R interval, RMSSD, root mean square of the standard deviation, VAS, visual analogue scale. mOFC, medial orbitofrontal cortex, PoCG, post-central gyrus, vCG, ventral cingulate gyrus. 


\section{FIGURES}

\begin{tabular}{|c|c|c|c|}
\hline 8.30 & $\begin{array}{l}\text { Urine osmolality } \\
\text { Body temperature } \\
\text { Pre-breakfast weight } \\
\text { Pre-breakfast thirst }\end{array}$ & \multirow{7}{*}{$\begin{array}{l}\text { Fluid loss } \\
\text { due to } \\
\text { perspiration } \\
\text { and } \\
\text { breathing. }\end{array}$} & \\
\hline 8.45 & $\begin{array}{l}\text { Standard breakfast } \\
\text { Post breakfast weight } \\
\text { Post breakfast thirst }\end{array}$ & & \multirow{6}{*}{$\begin{array}{l}\text { Total } \\
\text { amount } \\
\text { of weight } \\
\text { lost. }\end{array}$} \\
\hline 9.00 & $\begin{array}{l}\text { Memory, Attention, Mood } \\
\text { Heart rate variability }\end{array}$ & & \\
\hline 10.00 & $\begin{array}{l}\text { Rest } \\
\text { Drinks were provided at } \\
10.10 \text { and } 12.10\end{array}$ & & \\
\hline 12.40 & Memory, Attention, Mood & & \\
\hline 12.50 & $\begin{array}{l}\text { Body temperature } \\
\text { Thirst } \\
\text { Weight before urination }\end{array}$ & & \\
\hline 13.00 & $\begin{array}{l}\text { Urine osmolality } \\
\text { Weight after urination } \\
\text { Heart rate variability }\end{array}$ & & \\
\hline
\end{tabular}

\section{$13.15 \quad$ MRI procedure}

Figure 1. The experimental procedure. 


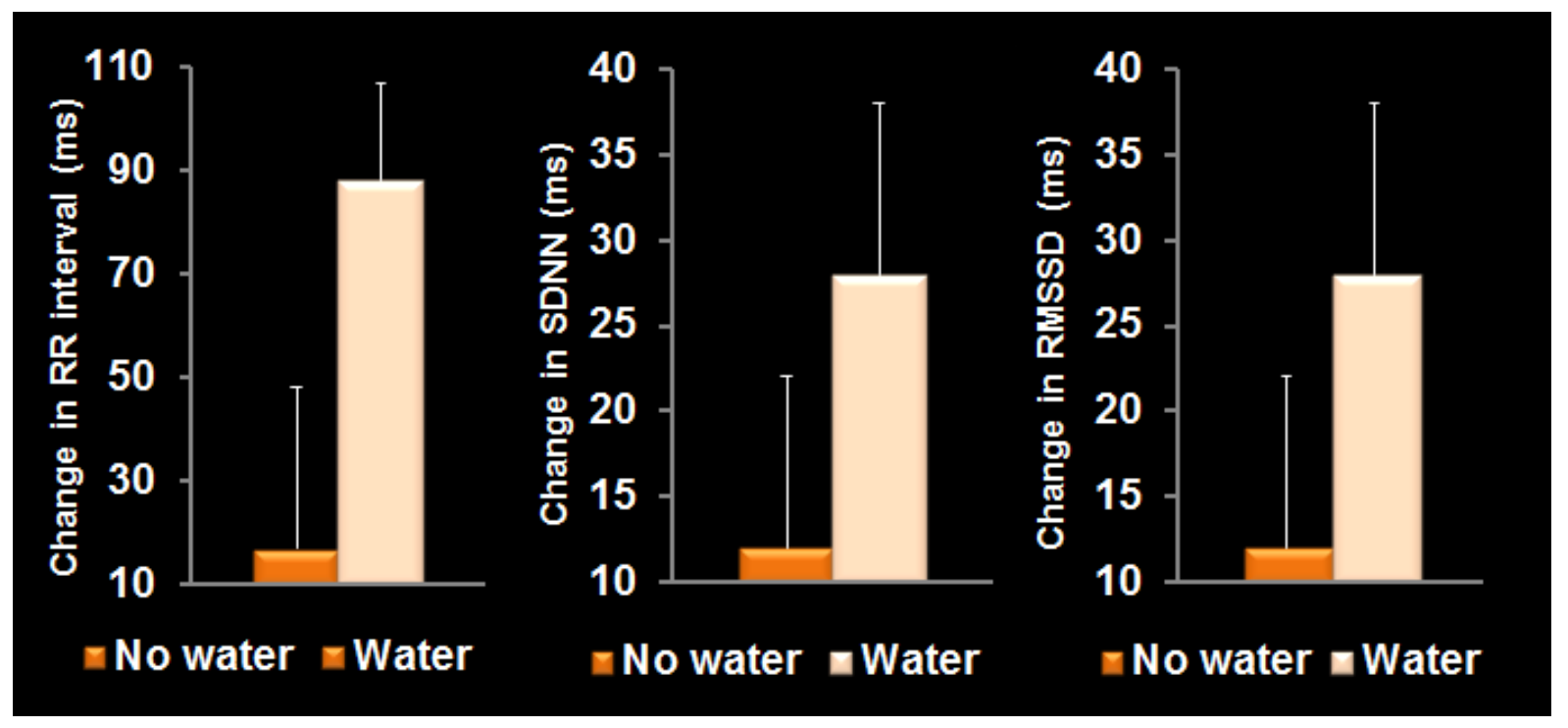

Figure 2. The effect of drinking water on the average length of the RR interval, SDNN and RMSSD.

Data are the changes (end of the morning minus baseline) for each index compared across the two conditions. When participants were hypo-hydrated they had a lower R-R (i.e a higher HR) $(p<0.03)$, lower SDRR (i.e a lower HRV) ( $p<0.01$ ) and reduced parasympathetic activity (i.e. lower RMSSD) $(p<0.03)$. RR, interbeat interval, SDNN, standard deviation of normal to normal R-R interval, RMSSD, root mean square of the standard deviation. 


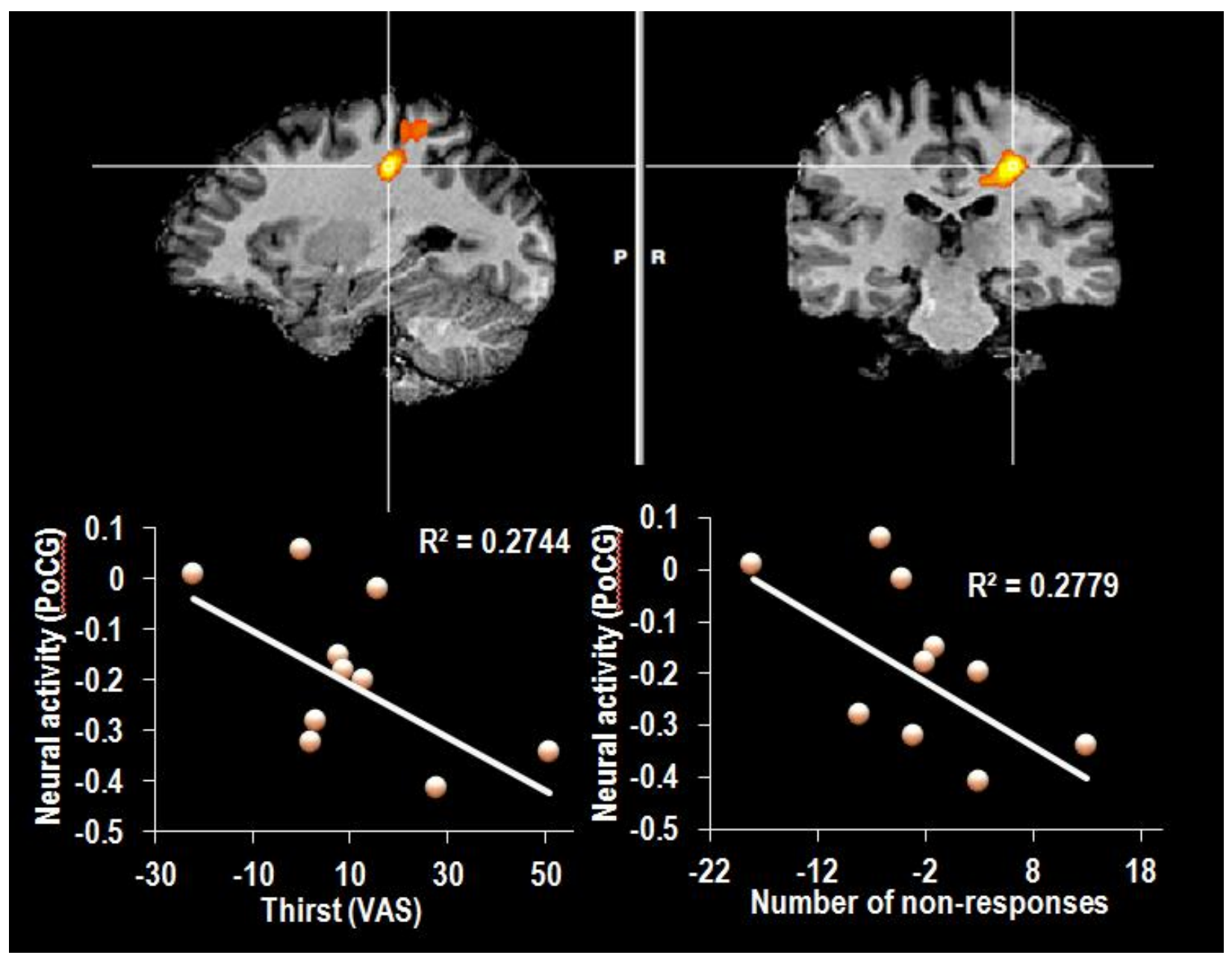

Figure 3. A. Peak activity in PoCG water > hypo-hydration. Correlation between neuronal activity in the PoCG and B. thirst and C. number of non-responses on the arithmetic task. Data are Pearson's $r$ coefficient for difference scores (no water - water). 


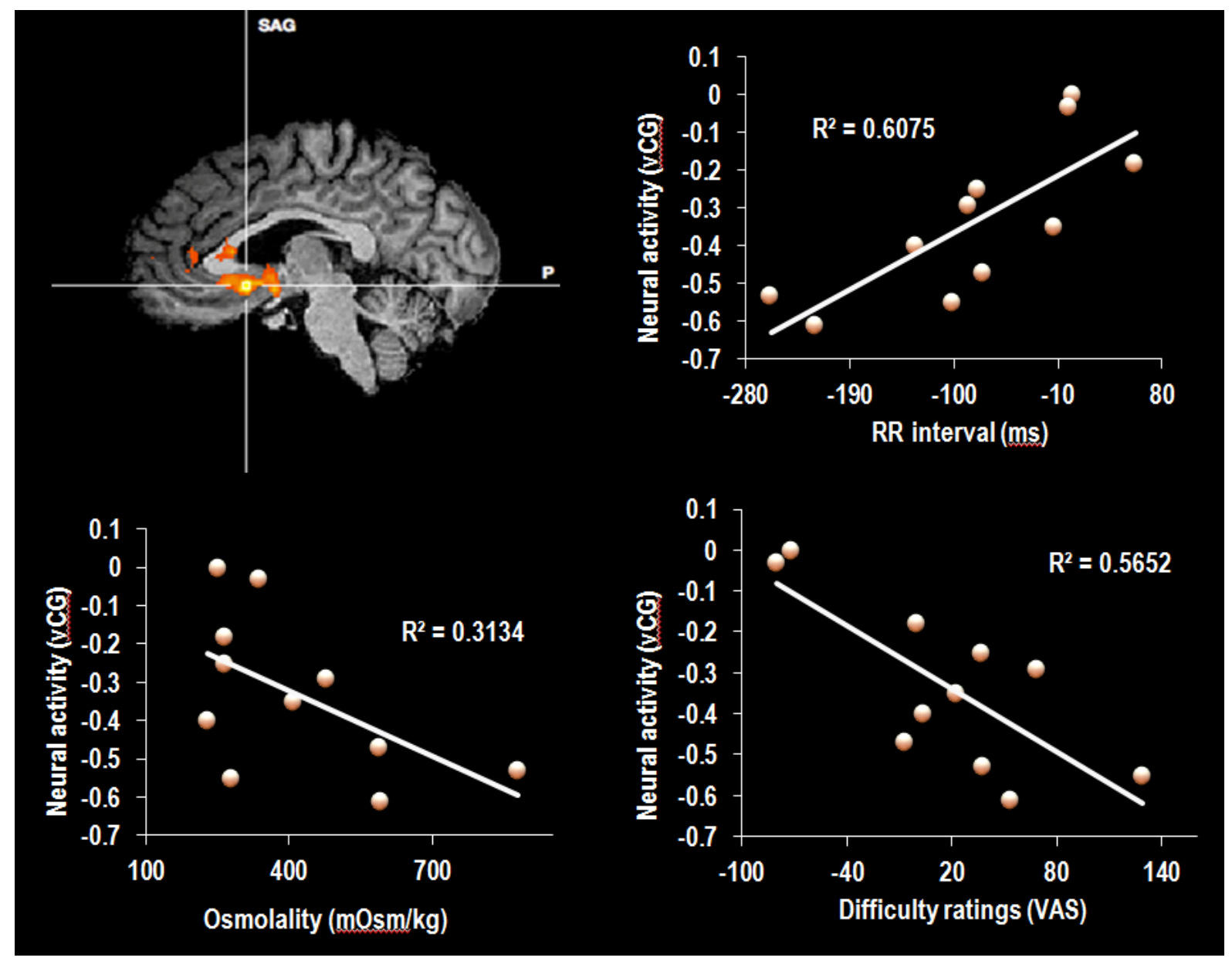

Figure 4. A. Peak activity in vCG water $>$ hypo-hydration. Correlation between neuronal activity in the VCG and B. RR interval C. osmolality and D. difficulty ratings. Data are Pearson's $r$ coefficient for difference scores (no water - water). 

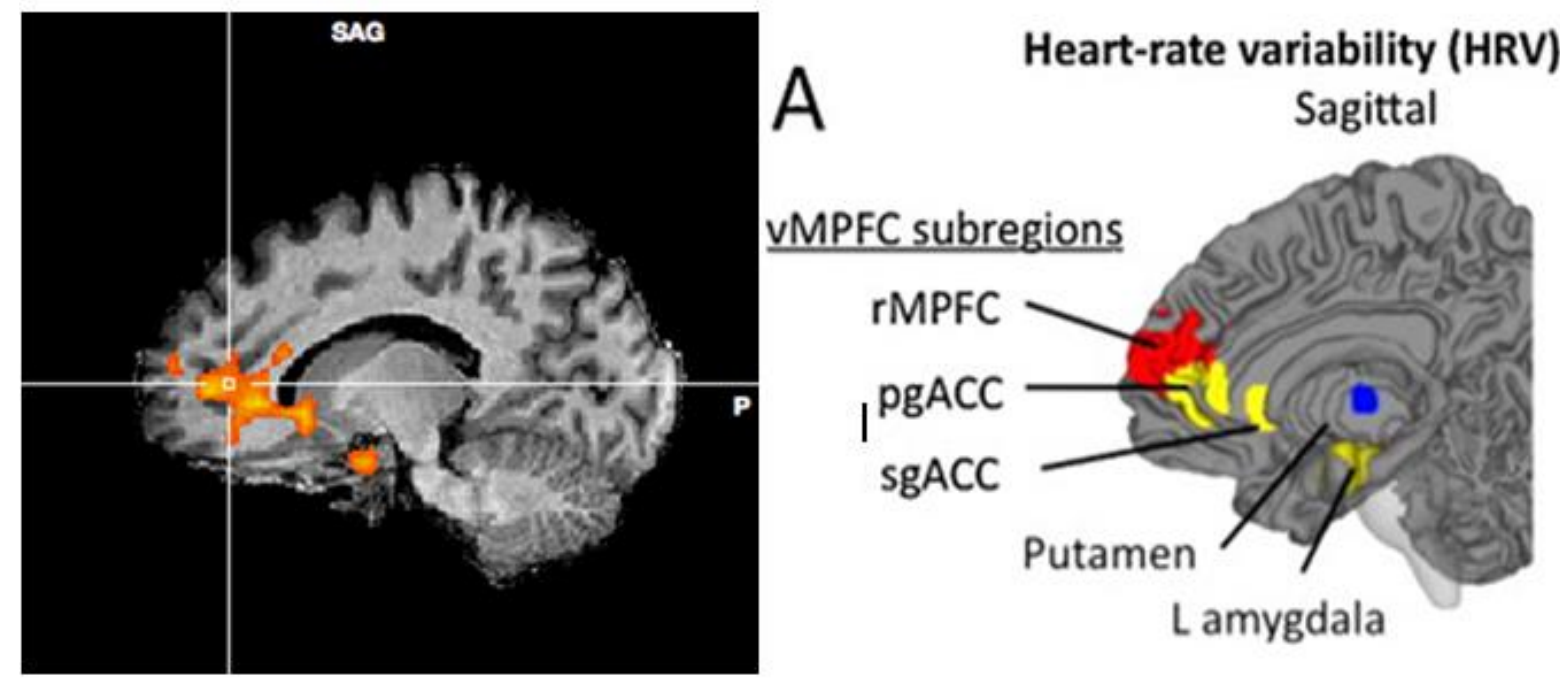

Figure 5. Map showing the brain areas associated with autonomic modulation.

Left data from present study. Right areas controlling HRV reproduced from Thayer et al. (2012) with permission. 


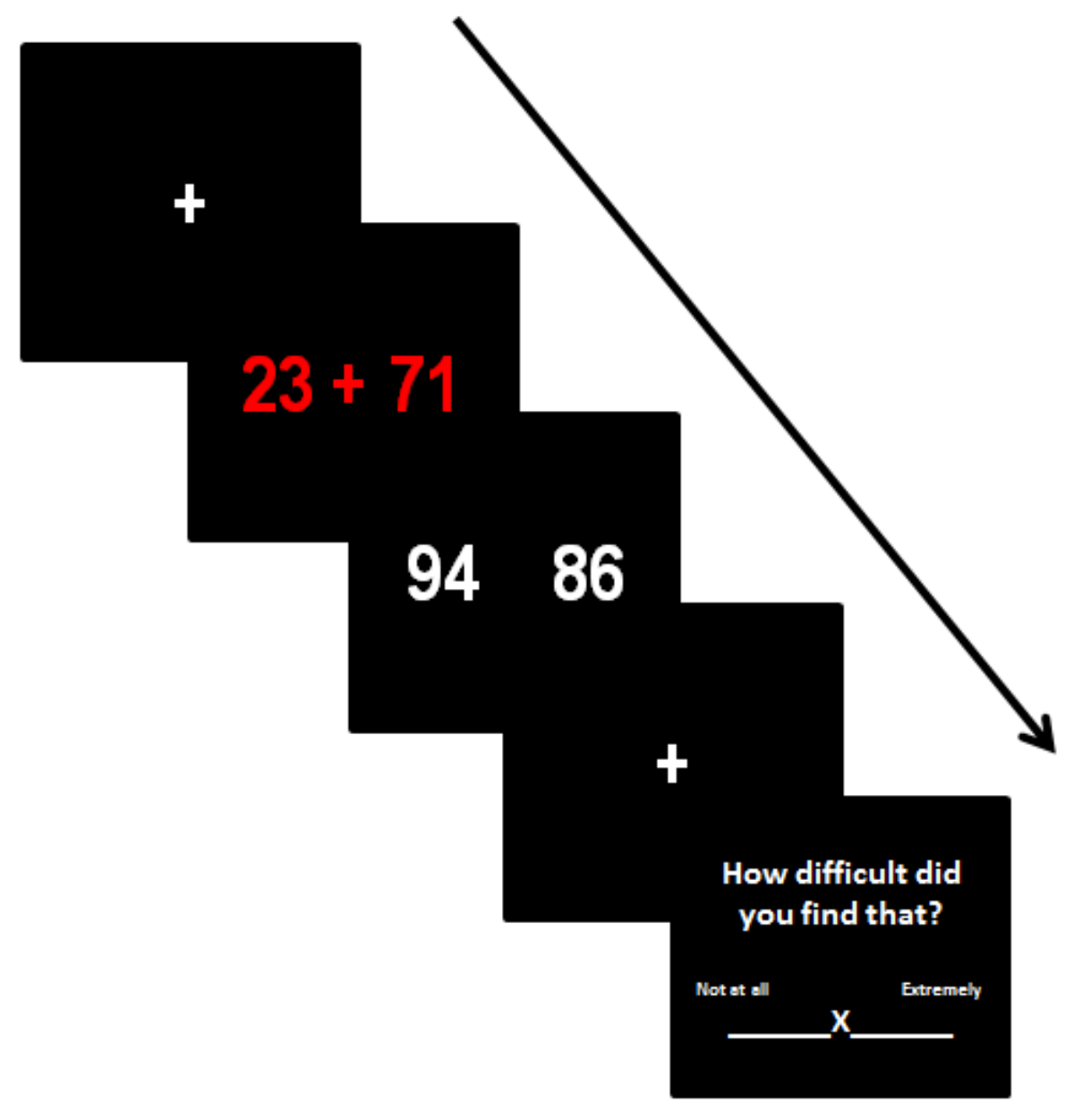

Figure 6. Arithmetic task and Visual analogue scale example stimuli.

The crosses are fixation points when otherwise the screen is blank. At the third image the participant indicated whether the right or left number was correct by pushing a button 
